\title{
Germline NLRP7 mutations: genomic imprinting and hydatidiform mole
}

\author{
Pei Hui ${ }^{1}$
}

Received: 5 March 2020 / Revised: 5 March 2020 / Accepted: 18 March 2020 / Published online: 23 June 2020

(C) Springer-Verlag GmbH Germany, part of Springer Nature 2020

Hydatidiform moles are abnormal gestations with trophoblastic proliferation and villous hydrops and are generally classified into complete and partial moles [1]. At the genetic level, the presence of excessive paternal genome plays a key role in the development of the disease, likely through altered genomic imprinting. Identification of specific gene mutations of $N R L P 7$ and $K H D C 3 L$ in recent decades in a subset of familial biparental complete moles (FBCHM) has provided insight to our understanding of pathogenesis of both sporadic moles and FBCHMs. In this issue of Virchows Archives, Allias and coauthors describe a 24-year-old woman who carried a novel germline NLRP7 truncating mutation and developed four consecutive gestations with abnormal chorionic villous phenotypes ranging from complete to partial mole-like changes [2]. The paper provides new evidence of interest.

The mammalian placenta is enriched with imprinted genes, many of which are functionally related to cellular proliferation and almost all imprinted genes that are specific to the placenta are paternally imprinted and functionally expressed only from the maternal alleles [3]. Based on the "parental conflict hypothesis" [4], the intent of the paternal genome is to maximize resources for the father's own progeny but the interest of the maternal genome is to distribute resources equally among her offspring, implying that growth-promoting genes are mainly expressed from the paternally inherited genome and are suppressed in the maternally inherited counterparts. Paternal-only or excess paternal genomic composition is the key genetic event underlying the development of sporadic moles. Sporadic complete moles inherit a diploid, androgenic-only nuclear genome of either homozygosity (up to $90 \%$ ) or heterozygosity $(10 \%)$. Partial moles have a triploid diandricmonogynic genome, harboring two heterozygous sperm haploid genomes in over $95 \%$ of the cases. Such excessive

Pei Hui

pei.hui@yale.edu

1 Department of Pathology, Yale University School of Medicine, 310 Cedar Street, New Haven, CT 06520, USA paternal genomic representation in sporadic moles likely disrupts the normal imprinting control leading to abnormal placental trophoblast proliferation [5]. Systemic evaluation of imprinting defects revealed that there was total paternalization of all ubiquitous and placenta-specific DMRs (differentially methylated regions) in sporadic androgenetic moles. Consistent with the "parental conflict interest" theory, the molar phenotypes are likely due to defective placenta-specific imprinting and abnormal overexpression of paternally imprinted gene transcripts.

FBCHM is an exceptional condition of recurrent complete moles in multiple pregnancies [6]. NLRP7 is a human maternal effect gene that is expressed in the oocyte to support embryonic development until the activation of embryonic genome occurs. Around 50 mutations of $N L R P 7$ gene are responsible for majority of FBCHM as a causal event. KHDC $3 L$ is the second maternal effect gene identified in $10-14 \%$ of FBCHM cases. Mutations in NLRP7 and KHDC3L could confer a complete molar phenotype on digynic triploid conceptions [7] and that normal egg donation enables FBCHM patients to achieve normal term pregnancy firmly establish a pivotal role of $N L R P 7$ and $K H D C 3 L$ in the pathogenesis of FBCHM [8].

NRLP7 plays an essential role in controlling the timing of oocyte growth or in transducing signals for the initiation of imprint establishment and works in a stage-dependent fashion during human preimplantation development. KHDC3L protein displays a juxta perinuclear signal and colocalizes with NLRP7 in human oocytes and preimplantation embryo. Although the genome of FBCHM is contributed by both parents, gene expression patterns are found similar to those seen in sporadic androgenetic complete moles [9]. Molar tissue of FBCHM of patients with either NLRP7 or KHDC $3 L$ mutations demonstrated imprinting gene defects with silencing of maternally imprinted genes and expression of paternally imprinted counterparts. It is plausible that $N L R P 7$ or $K H D C 3 L$ mutations result in defective placental-specific imprinting mechanism shutting down or reducing expression of maternally imprinted genes and overexpression of paternally derived transcripts. 
While $N L R P 7$ mutations are primarily described in FBCHM, depending on the severity of NLRP7 mutations, a subset of FBCHMs expressed variable levels of p57(KIP2) expression and showed histological features simulating diandric triploid partial moles and were closely associated with missense mutations. In contrast, cases of FBCHMs with abnormal p57(KIP2) expression and histological changes of complete mole were significantly associated with proteintruncating mutations [10]. This is further complicated by the observation in the current paper by Allias et al. that even an identical NLRP7 truncating mutation in the same patient presented with gestations of variable histological phenotypes and p57 staining patterns, ranging from complete to partial mole. P57 gene is a paternally imprinted gene encoding a cyclindependent kinase inhibitor and is located within the imprinting gene clusters on the short arm of chromosome 11 (11p15.4). In a closely related scenario, uniparental isodisomy is an abnormal genetic condition in which both homologous chromosomes or part of the chromosome are inherited from one parent and the other parent's homologous chromosome is lost. We recently described three cases of gestations with paternal uniparental isodisomy at tyrosine hydroxylase or THO1 (another paternally imprinted gene) locus on chromosome 11p15.4. The three cases demonstrated a spectrum of morphological changes and/or p57 immunohistochemical features simulating either partial or complete mole. One of the three patients even developed clinical complications simulating persistent gestational trophoblastic disease/neoplasia that required multiagent chemotherapy [11]. It is striking that, similar to FBCHM, paternal uniparental isodisomy at the THO1 locus (colocalizing with p57 at chromosome 11p15.4) results in abnormal gestations simulating hydatidiform moles both histologically and clinically and the degree to which it simulates a hydatidiform mole appears to depend on the extent of altered expression of paternally imprinted genes on chromosome $11 \mathrm{p} 15$.

Taken together, it can be hypothesized that the global imprinting alteration at a critical time point in a preimplantation embryo is acquired by either the de novo absence of the maternal haploid genome in sporadic androgenetic complete moles or mutations of $N L R P 7$ or $K D C 3 L$ leading to the suppression of the entire maternal imprinting gene expression in FBCHM. The findings in Allias's paper further emphasize a close and complex interplay between NLRP7 mutations, DNA methylation regulation, and imprinting gene expression, such as p57 in the oocytes that likely drives villous trophoblasts to acquire molar phenotypes in FBCHM. Future investigations into the role of NLRP7 mutations in FBCHM may ultimately elucidate the converging biological event that underscores the development of hydatidiform moles of both sporadic and familial biparental nature.

Compliance with ethical standards Dr. Pei Hui is the sole author of the work.

Conflict of interest The author declares no conflict of interest.

\section{References}

1. Hui P, Baergen R, Cheung A, Fukunaga M, Gersell D, Lage JM, Ronnett BM, Sebrie NJ, Wells M (2014) Gestational trophoblastic disease. In: Kurman R, Carcangiu ML, Herrington S, Young R (eds) WHO classification of tumours of female reproductive organs, 4th edn. International Agency for Research on Cancer, Lyon

2. Allias F, Mechtouf N, Gaillot-Durand L, Hoffner L, Hajri T, Devouassoux-Shisheboran M, Massardier J, Golfier F, Bolze PA, Surti U, Slim R (2020) A novel NLRP7 protein-truncating mutation associated with discordant and divergent $\mathrm{p} 57$ immunostaining in diploid biparental and triploid digynic moles. Virchows Arch:1-7. https://doi.org/10.1007/s00428-020-02769-w

3. Hui P (2012) Developmental biology of the placenta. In: Hui P (ed) Gestational trophoblastic disease-diagnostic and molecular genetic pathology, 1st edn. Humana Press/Springer

4. Haig D, Westoby M (2006) An earlier formulation of the genetic conflict hypothesis of genomic imprinting. Nat Genet 38(3):271

5. Devriendt K (2005) Hydatidiform mole and triploidy: the role of genomic imprinting in placental development. Hum Reprod Update 11(2):137-142

6. Nguyen NM, Slim R (2014) Genetics and epigenetics of recurrent hydatidiform moles: basic science and genetic counselling. Curr Obstet Gynecol Rep 3:55-64

7. Fallahian M, Sebire NJ, Savage PM, Seckl MJ, Fisher RA (2013) Mutations in NLRP7 and KHDC3L confer a complete hydatidiform mole phenotype on digynic triploid conceptions. Hum Mutat 34(2):301-308

8. Fisher RA, Lavery SA, Carby A et al (2011) What a difference an egg makes. Lancet 378(9807):1974

9. Fisher RA, Hodges MD, Rees $\mathrm{HC}$ et al (2002) The maternally transcribed gene p57(KIP2) (CDNK1C) is abnormally expressed in both androgenetic and biparental complete hydatidiform moles. Hum Mol Genet 11(26):3267-3272

10. Nguyen NM, Zhang L, Reddy R, Déry C, Arseneau J, Cheung A, Surti U, Hoffner L, Seoud M, Zaatari G, Bagga R, Srinivasan R, Coullin P, Ao A, Slim R (2014) Comprehensive genotypephenotype correlations between NLRP7 mutations and the balance between embryonic tissue differentiation and trophoblastic proliferation. J Med Genet 51(9):623-634

11. Buza N, McGregor SM, Barroilhet L, Zheng X, Hui P (2019) Paternal uniparental isodisomy of tyrosine hydroxylase locus at chromosome 11p15.4: spectrum of phenotypical presentations simulating hydatidiform moles. Mod Pathol 32(8):1180-1188

Publisher's note Springer Nature remains neutral with regard to jurisdictional claims in published maps and institutional affiliations. 